\title{
ON THE HOMOTOPY TYPE OF STIEFEL MANIFOLDS
}

\author{
I. M. JAMES
}

ABSTRACT. Suppose that we have a fibre bundle where the total space has the same homotopy type as the product of the fibre and the base. When can we conclude that the bundle is trivial, in the sense of fibre bundle theory? This question arises in the classification theory of Hopf homogeneous spaces, especially in relation to Stiefel manifolds. Results are proved, using cohomology operations, which answer the question in some cases.

1. Introduction. Let $n \geqq k \geqq 1$. As usual we denote by $V_{n, k}$ the real Stiefel manifold of orthonormal $k$-frames in euclidean $n$-space, and by $W_{n, k}$ (resp. $X_{n, k}$ ) the corresponding complex (resp. quaternionic) Stiefel manifold. We also, when convenient, use the notation $O_{n, k}$ to cover all three cases, as in [10]. Set $d=1,2$ or 4 according as the case is real, complex or quaternionic, so that $O_{n, 1}$ is a $(d n-1)$-sphere. Write $O_{n, 1}=S_{n}$ and consider the fibration $p: O_{n, k} \rightarrow S_{n}$ given by taking (say) the last vector in each $k$-frame to form a 1-frame. Excluding the case $k=1$ we identify the fibre with $O_{n-1, k-1}$ in the usual way. By (4.5) of [11] we have at once

THEOREM (1.1). If $O_{n, k}$ has the same homotopy type as $S_{n} \times O_{n-1, k-1}$ then $p$ admits a cross-section.

The main purpose of this note is to prove

ThEOREM (1.2). If $O_{n, k}$ has the same homotopy type as $S_{n} \times O_{n-1, k-1}$ then the interval $[n-k+1, n]$ contains a power of two.

Let us examine the implications of these results in each of the three cases. In the real case we recall, from [2], that $p: V_{n, k} \rightarrow S^{n-1}$ admits a cross-section if and only if

$$
n \equiv 0 \bmod 2^{\phi(k)} \text {, }
$$

where $\phi(k)$ denotes the number of integers $m$ in the range $0<m<k$ such that $m \equiv 0,1,2$ or $4 \bmod 8$. If (1.3) is satisfied then, for $k \geqq 2$, the interval $[n-k+1, n-1]$ does not contain a power of two. Hence (1.1) and (1.2) imply

Received by the editors June $17,1970$.

AMS 1970 subject classifications. Primary 55D15; Secondary 55E15, 55F10, 55G05, 55G20, 55F20.

Key words and phrases. Stiefel manifold, homotopy type, cross-section, Steenrod square, secondary cohomology operation, Whitehead product, Hopf construction, Hopf homogeneous space, fibration. 
THEOREM (1.4). Let $n \geqq k \geqq 2$. If $V_{n, k}$ has the same homotopy type as $S^{n-1} \times V_{n-1, k-1}$ then $n=2^{r}$ for some integer $r \geqq \phi(k)$.

The proof of (1.2) depends on the relations of Adem [3] between the Steenrod squares. Another result can be obtained by using the secondary cohomology operations of Adams [1]. We shall prove

THEOREM (1.5). Let $n \geqq k \geqq 2$ and let $k$ be even. If $V_{n, k}$ has the same homotopy type as $S^{n-1} \times V_{n-1, k-1}$ then $n=2,4$ or 8 .

A proof of (1.5) for the case $k=2$ is given in [12], modulo the main result of [1]. Note that the fibration is trivial when $n=2,4$ or 8 . It is tempting to conjecture that (1.5) is true for all values of $k$. This would be an important step towards the classification of Hopf homogeneous spaces (see [8]).

Let $w_{r} \in \pi_{2 r-1}\left(S^{r}\right)(r=1,2, \cdots)$ denote the Whitehead square of the generator $\iota_{r} \in \pi_{r}\left(S^{r}\right)$. It is a standard problem, as yet unsolved, to determine the values of $r$ such that $w_{r}$ can be halved, i.e. such that $w_{r} \in 2 \pi_{2 r-1}\left(S^{r}\right)$. We shall prove

THEOREM (1.6). If $V_{n, 3}$ has the same homotopy type as $S^{n-1} \times V_{n-1,2}$ then $w_{n-1}$ can be halved.

It is known that $w_{15}$ can be halved but, so far as I am aware, it is not known whether $V_{16,3}$ has the same homotopy type as $S^{15} \times V_{15,2}$.

Thus our results are not conclusive in the real case, but in the complex and quaternionic cases we obtain

Theorem (1.7). Let $n \geqq k \geqq 2$, but exclude $n=k=2$. Then $W_{n, k}$ does not have the same homotopy type as $S^{2 n-1} \times W_{n-1, k-1}$.

THEOREM (1.8). Let $n \geqq k \geqq 2$. Then $X_{n, k}$ does not have the same homotopy type as $S^{4 n-1} \times X_{n-1, k-1}$.

Suppose that $p: W_{n, k} \rightarrow S^{2 n-1}$ has a cross-section. Then $2 n \equiv 0$ $\bmod 2^{\phi(2 k)}$, by (1.3), since $p: V_{2 n, 2 k} \rightarrow S^{2 n-1}$ has a cross-section. When $k \geqq 3$, however, it is shown in $\$ 20$ of [5] that $n$ is not a power of two, and hence the interval $[n-k+1, n]$ does not contain a power of two. Thus (1.7) follows from (1.1) and (1.2) when $k \geqq 3$. The case $k=2$ has already been dealt with in [12]. The proof of (1.8) is similar except that the case $k=2$ does not require separate consideration.

2. Construction of a complex. Throughout this note we work in the category of pointed spaces using cohomology with mod 2 coefficients. Let $K$ be a $(q-1)$-connected complex, where $q>0$. Suppose that we have an element $\xi \in \pi_{q}(K)$ such that the homomorphism 


$$
\xi^{*}: H^{q}(K) \rightarrow H^{q}\left(S^{q}\right)
$$

is nontrivial. Let $m>q$ and suppose that there exists an element $\gamma \in \pi_{m+q-1}\left(S^{q}\right)$ such that

$$
\text { (a) } \xi \circ \gamma=0, \quad \text { (b) } S^{m-q} \gamma=w_{m},
$$

where $S$ denotes the suspension functor. Write $K^{\prime}=S^{m-q} K$ and write $\xi^{\prime}=S^{m-q \xi}$, so that $\xi^{\prime} \in \pi_{m}\left(K^{\prime}\right)$. It follows at once from (2.1) that $\left[\xi^{\prime}, \xi^{\prime}\right]=\xi^{*} w_{m}=0$, and hence that there exists a map

$$
\theta: S^{m} \times S^{m} \rightarrow K^{\prime}
$$

of type $\left(\xi^{\prime}, \xi^{\prime}\right)$. Let $\phi: S^{2 m+1} \rightarrow S K^{\prime}$ be obtained by performing the Hopf construction on $\theta$, and let

$$
L=e^{2 m+2} \cup S K^{\prime}
$$

denote the mapping cone of $\phi$. We have the cohomology exact sequence

$$
H^{r}\left(L, S K^{\prime}\right) \stackrel{i^{*}}{\rightarrow} H^{r}(L) \stackrel{j^{*}}{\rightarrow} H^{r}\left(S K^{\prime}\right) .
$$

Let $\lambda \in H^{2 m+2}(L)$ denote the image under $i^{*}$ of the generator of the relative group. By assumption there exists an element $\sigma \in H^{q}(K)$ such that $\xi^{*} \sigma \neq 0$. Hence $\xi^{*} \sigma^{\prime} \neq 0$, where $\sigma^{\prime} \in H^{m}\left(K^{\prime}\right)$ is the iterated suspension of $\sigma$. Hence it follows from (1.4) of [14] that

$$
\lambda=\mu \cup_{\mu}=S q^{m+1} \mu,
$$

where $\mu \in H^{m+1}(L)$ is the element such that $j^{*} \mu=S^{*} \sigma^{\prime}$. We need a condition to ensure $\lambda \neq 0$, such as

Lemma (2.3). Suppose that $H^{m+q}(K)$ is spanned by decomposable elements. Then $L$ can be constructed, by suitable choice of $\theta$, so that $\lambda \neq 0$.

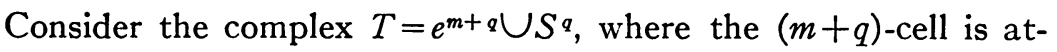
tached by means of $\gamma$. By (2.1a) there exists a map $t: T \rightarrow K$ such that $t \mid S^{q}$ represents $\xi$. Now $T$ has trivial cup-products since $m>q$. Hence

$$
t^{*}: H^{m+q}(K) \rightarrow H^{m+q}(T)
$$

is trivial, and hence so is

$$
\left(S^{m-q} t\right)^{*}: H^{2 m}\left(S^{m-q} K\right) \rightarrow H^{2 m}\left(S^{m-q} T\right) .
$$

By (2.1b) the $2 m$-cell of $S^{m-q} T=e^{2 m} \cup S^{m}$ is attached by the White- 
head square $w_{m}$. Hence we can regard $S^{m-q} T$ as obtained from $S^{m} \times S^{m}$ by identifying axes in the usual way. Take $\theta=S^{m-q} \circ \circ \psi$, where $\psi$ : $S^{m} \times S^{m} \rightarrow S^{m-q} T$ is the identification map. Write $S^{m-q} K=K^{\prime}$, as before. Then

$$
\theta^{*}: H^{2 m}\left(K^{\prime}\right) \rightarrow H^{2 m}\left(S^{m} \times S^{m}\right)
$$

is trivial and hence so is

$$
\phi^{*}: H^{2 m+1}\left(S K^{\prime}\right) \rightarrow H^{2 m+1}\left(S^{2 m+1}\right) .
$$

Hence it follows at once from exact sequence considerations that $\lambda \neq 0$, as asserted.

Now suppose that $K$ contains a sub-complex $M$, with inclusion $l: M \rightarrow K$, such that $M$ is an $S$-retract of $K$. Suppose that $\xi=l_{*} \eta$, for some element $\eta \in \pi_{q}(M)$, and hence $\xi^{*} \sigma=\eta^{*} l^{*} \sigma$. Choose $p$, where $p>m-q$, such that there exists a retraction $\rho: S^{p} K \rightarrow S^{p} M$. Consider the complex $N=e^{p+q+m+1} \cup S^{p} M$ which is obtained from $S^{p+q-m-1} L$ by identifying points of $S^{p} K$ with their images under $\rho$. With all these hypotheses, including (2.1), we find that (2.2) and (2.3) imply

Lemma (2.4). Suppose that $H^{m+q}(K)$ is spanned by decomposable elements. Then there exists a complex $N=e^{p+q+m+1} \cup S^{p} M$, for sufficiently large values of $p$, such that

$$
S q^{m+1}: H^{p+q}(N) \rightarrow H^{p+q+m+1}(N)
$$

is nontrivial.

After some preliminaries of a different kind we apply (2.4), in $\$ 4$ below, to establish the main results stated in the introduction.

3. The Whitehead product. Let $p: E \rightarrow S^{m}(m \geqq 2)$ be a fibration and let $v: F \rightarrow E$ denote the inclusion of the fibre. Suppose that there exists a homotopy equivalence $h: S^{m} \times F \rightarrow E$. By restricting $h$ to the axes of the product we obtain a pair of maps

$$
S^{m} \stackrel{f}{\rightarrow} E \stackrel{g}{\leftarrow} F .
$$

Let $\alpha \in \pi_{m}(E)$ be the class of $f$. We prove

Lemma (3.1). If $\beta \in \pi_{r}(E)$, where $r<m$, then the Whitehead product $[\alpha, \beta]$ is zero.

Identify $\pi_{*}\left(S^{m} \times F\right)$ with $\pi_{*}\left(S^{m}\right) \oplus \pi_{*}(F)$ in the standard way, so that $h_{*}$ is transformed into

$$
\left(f_{*}, g_{*}\right): \pi_{*}\left(S^{m}\right) \oplus \pi_{*}(F) \rightarrow \pi_{*}(E) .
$$


Since $r<m$ we have $\beta=g_{*} \mu$, say, where $\mu \in \pi_{r}(F)$. Also $\alpha=f_{*} \lambda$, by definition, where $\lambda$ generates $\pi_{m}\left(S^{m}\right)$. Choose a homotopy inverse $h^{\prime}: E \rightarrow S^{m} \times F$ of $h$. Since $h_{*}^{\prime} f_{*} \lambda=(\lambda, 0)$ and $h_{*}^{\prime} g_{*} \mu=(0, \mu)$ it follows by naturality that

$$
h_{*}^{\prime}\left[f_{* \lambda}, g_{* \mu}\right]=([\lambda, 0],[0, \mu])=0,
$$

in $\pi_{*}\left(S^{m} \times F\right)$. Hence $\left[f_{*} \lambda, g_{*} \mu\right]=0$, in $\pi_{*}(E)$, since $h^{\prime}$ is a homotopy equivalence. This proves (3.1).

Next we prove (cf. (4.5) of [11]) under the same preliminary hypothesis

Lemma (3.2). Suppose that $\pi_{m}(F)$ is finite and that $\pi_{m-1}(F)$ is finitely generated. Then $p f: S^{m} \rightarrow S^{m}$ is a homotopy equivalence.

We have $\pi_{m-1}(F) \approx \pi_{m-1}(E)$, since $\pi_{m-1}\left(S^{m}\right)=0$. Also $v_{*} \pi_{m-1}(F)$ $=\pi_{m-1}(E)$ and so, since $\pi_{m-1}(F)$ is finitely-generated, it follows that

$$
v_{*}: \pi_{m-1}(F) \approx \pi_{m-1}(E) \text {. }
$$

Hence $p_{*} \pi_{m}(E)=\pi_{m}\left(S^{m}\right)$, from the homotopy exact sequence. But $p_{*} g_{*} \pi_{m}(F)=0$, since $\pi_{m}(F)$ is finite and $\pi_{m}\left(S^{m}\right)$ is free. Hence $p_{*} f_{*} \pi_{m}\left(S^{m}\right)=\pi_{m}\left(S^{m}\right)$, and we obtain (3.2).

4. Proof of the theorems stated in the introduction. We now return to the situation described in $\$ 1$. Recall that $d=1,2$ or 4 according as the case is real, complex or quaternionic. Let $O_{r}(r=1,2, \cdots)$ denote the group of automorphisms of the appropriate $r$-space, so that $\left(O_{r}, d\right)=(O(r), 1),(u(r), 2)$ or $(\mathrm{Sp}(r), 4)$. We regard $O_{n}$ in the usual way, as a principal $O_{n-k}$-bundle over $O_{n, k}$. Consider the composition

$$
\pi_{d n-1}\left(O_{n, k}\right) \underset{\Delta}{\rightarrow} \pi_{d n-2}\left(O_{n-k}\right) \underset{J}{\rightarrow} \pi_{2 d n-d k-3}\left(S_{n-k}\right),
$$

where $\Delta$ denotes the transgression homomorphism, in the associated homotopy exact sequence, and $J$ is given by the Hopf construction, using the pointed action of $O_{n-k}$ on $S_{n-k}$. Suppose that there exists a cross-section $f: S_{n} \rightarrow O_{n, k}$. By (4.3) of [9] we have

$$
S^{d k-d} J \Delta \alpha= \pm w_{d n-1}
$$

where $\alpha$ denotes the class of $f$. We regard $O_{n, k}$, as before, as a bundle over $S_{n}$ with fibre $O_{n-1, k-1}$ but now, using $f$, reduce the group of the bundle to $O_{n-k}$. Consider the associated sphere-bundle $E$, with fibre $S_{n-k+1}$, as shown in the following diagram, where all the maps are inclusions. 


$$
\begin{array}{cc}
S_{n-k+1} \stackrel{u}{\rightarrow} E \\
i \downarrow \quad \downarrow j \\
O_{n-1, k-1} \underset{v}{\rightarrow} O_{n, k}
\end{array}
$$

By general theory we have a cross-section $q: S_{n} \rightarrow E$ such that $f=j q$. If $\sigma_{r}$ denotes the class of the identity on $S_{r}$ then

$$
\left[q_{*} \sigma_{n}, u_{*} \sigma_{n-k+1}\right]= \pm u_{*} J \Delta \alpha,
$$

by (3.7) of [12], and hence

$$
[\alpha, \xi]= \pm v_{*}(\xi \circ J \Delta \alpha),
$$

by naturality, where $\xi=i_{*} \sigma_{n-k+1}$ is a generator of $\pi_{d(n-k+1)-1}\left(O_{n-1, k-1}\right)$.

All the homotopy groups of $O_{n-1, k-1}$ are finitely-generated. Also $\pi_{d n-1}\left(O_{n-1, k-1}\right)$ is finite provided we assume, in the real case, that

$$
n \geqq \max (2 k-1,5) \text {. }
$$

Using the results of $\$ 3$ we prove

Lemma (4.4). Suppose that $O_{n, k}$ has the same homotopy type as $S_{n} \times O_{n-1, k-1}$. Also let (4.3) be satisfied in the real case. Then $\xi \circ J \Delta \alpha=0$ where $\alpha \in \pi_{d n-1}\left(O_{n, k}\right)$ is the class of a cross-section and $\xi$ is a generator, of $\pi_{d(n-k+1)-1}\left(O_{n-1, k-1}\right)$.

Let $f: S_{n} \rightarrow O_{n, k}$ be as in (3.2). Take $\alpha= \pm\{f\}$, whichever is the class of a cross-section. Then $[\alpha, \xi]=0$, by $(3.1)$; hence $v_{*}(\xi \circ J \Delta \alpha)$ $=0$, by (4.2). Since $v_{*}$ is injective this proves (4.4).

Let $Q_{n-1, k-1} \subset O_{n-1, k-1}$ denote the stunted quasi-projective space, as defined in [10]. We recall that $H^{r}\left(Q_{n-1, k-1}\right)=Z_{2}$ for $r=q d-1$ $(q=n-k+1, \cdots, n-1)$ while otherwise $H^{*}\left(Q_{n-1, k-1}\right)$ is trivial. Furthermore, $Q_{n-1, k-1}$ is an $S$-retract of $O_{n-1, k-1}$, as shown in (2.5) of [10]. To prove (1.2) we apply (2.4) with $(K, M)=\left(O_{n-1, k-1}, Q_{n-1, k-1}\right)$. In the real case we can assume (4.3) since otherwise (1.2) is trivial. If $O_{n, k}$ has the same homotopy type as $S_{n} \times O_{n-1, k-1}$ then $\gamma=J \Delta \alpha$ satisfies (2.1), by (4.1) and (4.4). Hence, by (2.4), there exists a complex

$$
N=e^{p+d(2 n-k+1)-1} \cup S^{p} Q_{n-1, k-1},
$$

for sufficiently large $p$, such that

$$
S q^{d n}: H^{p+d(n-k+1)-1}(N) \rightarrow H^{p+d(2 n-k+1)-1}(N)
$$

is nontrivial. Now $H^{p+r-1}(N)=0$ for $d n \leqq r<d(2 n-k+1)$. Hence, by 
the relations of Adem [3], the interval $[d(n-k+1), d n]$ contains a power of two, and so (1.2) is obtained.

In (1.5) we are concerned only with the real case, where $d=1$. We proceed as before and construct

$$
N=e^{p+2 n-k} \cup S^{p} Q_{n-1, k-1}
$$

such that $S q^{n}$ is nontrivial. By hypothesis $k$ is even, hence $n-k$ is even, and hence

$$
S q^{1}: H^{p+n-k}(N) \rightarrow H^{p+n-k+1}(N)
$$

is trivial. Let $N^{\prime}$ be a dual complex of $N$, in the sense of $S$-theory, so that, for an appropriate choice of $q$, we have that $H^{q-s}\left(N^{\prime}\right)=Z_{2}$ for $s=0,1, \cdots, k-2$ and for $s=n$, while otherwise $H^{*}\left(N^{\prime}\right)$ is trivial. By duality (see [13]) we have that

$$
S q^{i}: H^{q-i}\left(N^{\prime}\right) \rightarrow H^{q}\left(N^{\prime}\right)
$$

is trivial for $i=1$, nontrivial for $i=n$. By (1.3) we have $n=2^{r}$, where $r \geqq \phi(k)$. If $r \geqq 4$ then $k \leqq 2^{r-2}+2^{r-3}+1$ and we have direct contradiction with the main theorem of Adams [1] on secondary cohomology operations. Therefore $r \leqq 3$, as asserted.

It only remains for us to give the proof of (1.6), which concerns the real case when $k=3$. Let $n>4$, since otherwise (1.6) is trivial, and suppose that $V_{n, 3}$ has the same homotopy type as $S^{n-1} \times V_{n-1,2}$. Then $n \equiv 0 \bmod 4$, by (1.1) and (1.3). Consider (4.1) and (4.4), where in this case $J \Delta \alpha \in \pi_{2 n-5}\left(S^{n-3}\right)$. By exactness (4.4) implies that $J \Delta \alpha$ lies in the image of the transgression operator

$$
\Delta: \pi_{2 n-4}\left(S^{n-2}\right) \rightarrow \pi_{2 n-5}\left(S^{n-3}\right)
$$

in the homotopy exact sequence of the fibration $V_{n-1,2} \rightarrow S^{n-2}$. Since $n \equiv 0 \bmod 4$ we have $\pi_{2 n-4}\left(S^{n-2}\right)=S \pi_{2 n-5}\left(S^{n-3}\right)$, by the EHP sequence and (4.1) of [7]. Also $\Delta S=c_{*}$, where $c: S^{n-3} \rightarrow S^{n-3}$ is a map of degree 2. Hence $\Delta J \alpha=c_{*} \beta$, for some $\beta \in \pi_{2 n-5}\left(S^{n-3}\right)$, and hence $S^{2} c_{*} \beta=S^{2} J \Delta \alpha$ $=w_{n-1}$, by (4.1). Since $S^{2} c_{*} \beta=\left(S^{2} c\right)_{*} S^{2} \beta=2 S^{2} \beta$ this proves (1.6).

In conclusion I wish to thank Professor P. E. Thomas for some helpful comments on this work and on an alternative but slightly more complicated version in which $K$-theory is used instead of cohomology.

\section{REFERENCES}

1. J. F. Adams, On the non-existence of elements with Hopf invariant one, Ann. of Math. (2) 72 (1960), 20-104. MR 25 \#4530.

2. - Vector fields on spheres, Ann. of Math. (2) 75 (1962), 603-632. MR 25 \#2614. 
3. J. Adem, The iteration of the Steenrod squares in algebraic topology, Proc. Nat. Acad. Sci. U.S.A. 38 (1952), 720-726. MR 14, 306.

4. M. F. Atiyah, Thom complexes, Proc. London Math. Soc. (3) 11 (1961), 291310. MR 24 \#A1727.

5. A. Borel and J.-P. Serre, Groupes de Lie et puissances réduites de Steenrod, Amer. J. Math 75 (1953), 409-448. MR 15, 338.

6. M. Gilmore, Some Whitehead products on odd spheres, Proc. Amer. Math. Soc. 20 (1969), 375-377. MR 38 \#2777.

7. P. J. Hilton and J. H. C. Whitehead, Note on the Whitehead product, Ann. of Math. (2) 58 (1953), 429-442. MR 15, 642.

8. Wu-Yi Hsiang and J. C. Su, On the classification of transitive effective actions on Stiefel manifolds, Trans. Amer. Math. Soc. 130 (1968), 322-336. MR 36 \#4581.

9. I. M. James, On the iterated suspension, Quart. J. Math. Oxford Ser. (2) 5 (1954), 1-10. MR 15, 891.

10. —, Spaces associated with Stiefel manifolds, Proc. London Math. Soc. (3) 9 (1959), 115-140. MR 21 \#1596.

11. I. M. James and J. H. C. Whitehead, Note on fibre spaces, Proc. London Math. Soc. (3) 4 (1954), 129-137. MR 15, 892.

12. - The homotopy theory of sphere bundles over spheres. I, Proc. London Math. Soc. (3) 4 (1954), 196-218. MR 15, 892.

13. R. Thom, Espaces fibrés en sphères et carrés de Steenrod, Ann. Sci. Ecole Norm. Sup. (3) 69 (1952), 109-182. MR 14, 1004.

14. E. Thomas, On functional cup-products and the transgression operator, Arch. Math. 12 (1961), 435-444. MR 26 \#6972.

Oxford University Mathematical Institute, Oxford, England 